\title{
Treatment planning for the lung cancer
}

\author{
Sukhdeep Gill \\ Department of Radiation Oncology, University of Toledo, Toledo, Ohio, USA.
}

Received February 13, 2014; Revised March 10, 2014; Accepted March 12, 2014; Published Online March 16, 2014

\section{Letter to editor}

Volumetric modulated arc therapy (VMAT) and intensity modulated radiation therapy (IMRT) are commonly used treatment techniques for cancer treatment, and both the VMAT and IMRT techniques use the photon beam (mega-voltage X-rays) to deliver radiation dose to the tumor. The capability of modulating radiation beam has increased the ability of delivering more conformal dose distributions to tumor volume while minimizing dose to the normal tissues. The VMAT planning generally involves one or multiple arcs with gantry rotating around the patient, whereas the IMRT planning involves multiple static beams.

Current literature on radiation therapy for the lung cancer shows the publication of good number of dosimetric studies, which are typically focused on the treatment planning techniques ${ }^{1-7}$ and dose calculation algorithms. ${ }^{8-16}$ The treatment planning studies comparing the dosimetric quality between the IMRT and VMAT, however, do not provide the definitive conclusion, especially for the normal lung tissue. For example, Verbakel et al. ${ }^{1}$ performed the dosimetric study on 14 lung cancer cases and compared the IMRT and VMAT plans. It was reported that the V5 of the normal lung tissue was higher in the VMAT plans than in the IMRT plans. Ong et al. ${ }^{2}$ and Jiang et al. ${ }^{3}$ also reported higher V5 of the lung in the VMAT plans. The V5 of the lung from these studies ${ }^{1-3}$ show that the IMRT could be better at sparing the lung than the VMAT. However, the VMAT plans could produce lower values for the lung if dosimetric evaluation is done using different parameters. In the study by Jiang et al. ${ }^{3}$, normal lung was also evaluated for the mean dose, V20, and V30, and the results showed lower values in the VMAT plans than in the IMRT plans. ${ }^{3}$ This leads to the question- which dosimetric parameter for the normal lung tissue is more important when plan evaluation is done?

Corresponding author: Sukhdeep Gill; Department of Radiation Oncology, University of Toledo, Toledo, Ohio, USA.

Cite this article as:

Gill S. Treatment planning for the lung cancer. Int J Cancer Ther Oncol 2014; 2(1):020117. DOI: 10.14319/ijcto.0201.17
In addition to the sparing of normal tissues, the target coverage and dose homogeneity within the target volume are also equally important in radiation therapy. Jiang et al. ${ }^{3}$ found that the VMAT could produce better target coverage when compared to the IMRT. However, Rao et al. ${ }^{4}$, who performed study on 8 lung cases, found comparable target coverage in the VMAT and IMRT plans. Dose to the target volume was found to be similar in the VMAT and IMRT plans..$^{3-5}$

The variation in the reported results from various studies on lung cancer ${ }^{1-7}$ can be attributed to different factors such as plan optimization technique, experience of the treatment planner, treatment volume margins, dose prescription, location of the tumor, and dose calculation algorithms. The treatment plan optimization interface within the treatment planning system can let the user to assign weightings and objectives for the target and normal tissues. The final dosimetric results in the treatment plan may depend on the selection of weightings and objectives for the structures. Additionally, the plan optimization can be repeated with same set of optimization parameters or different ones if the dosimetric results have deviation from the compliance criteria.

Dose calculation algorithm incorporated in the treatment planning system can also affect the dosimetric results of the treatment plans, especially for the lung cancer, which includes the low-density medium. When radiation beam traverses the human body before it reaches the tumor, tissues of different electron density are encountered in the beam path. Presence of heterogeneity along the beam path may change the dose contribution to the tumor when compared to the homogeneous geometry. Such situation requires the dose calculation algorithm to consider the tissue heterogeneity correction when dose computations are performed on the cancer treatment plans.

Recent literature shows that the Monte Carlo based dose calculation algorithm is more appropriate for dose computations in the lung plans. ${ }^{8-10}$ Several investigators compared the analytical anisotropic algorithm (AAA) with the most recent algorithm called Acuros XB for the lung plans ${ }^{9-16}$, and vali- 
dation studies on Acuros XB show its superiority over the AAA, especially in inhomogeneous media. ${ }^{8-9,11,17-18}$ The beam modeling within the Acuros XB algorithm considered to be based on the Monte Carlo approach. ${ }^{18}$

The literature comparing the $\mathrm{AAA}$ and Acuros $\mathrm{XB}$ in the lung plans showed that the Acuros $\mathrm{XB}$ could produce higher values for the V20 ${ }^{11,12}$ and V5. ${ }^{10-12}$ If the Acuros XB is considered to be more accurate than the AAA, does the AAA underestimate the lung dose? It was also reported that the higher number of monitor units (MUs) will be required for the Acuros $\mathrm{XB}$ in order to achieve the target coverage similar to that of the AAA. ${ }^{9}$ The decreased target coverage using Acuros XB for the same number of MUs as in the AAA plans may not be clinically acceptable. If the treatment planning systems have an option to normalize the plan (e.g., target volume receiving certain percentage of the prescription dose), the Acuros XB plans can be normalized to achieve the desired target coverage, but such method may also increase the MUs, and this will increase the normal tissue dose and hot spot. Hence, treatment plans computed with different dose calculation algorithms are likely to provide different dosimetric results. Treatment plans calculated with different beam energy may also produce difference dosimetric results. ${ }^{19,20}$

Although there are uncertainties in the dosimetric results between the IMRT and VMAT plans, the common agreement among different published studies ${ }^{1-7}$ is the decreased delivery time and a smaller number of MUs using the VMAT than using the IMRT. Clinical trials comparing the IMRT and VMAT may be more helpful in establishing superiority of one technique over another. Multi-institutional study using the same dataset, beam parameters, and dose calculation algorithms/treatment planning system would help in reducing the uncertainties in the dosimetric results of the lung treatment plans. Studies based on the radiobiological models in the treatment planning could also be beneficial for more accurate prediction of tumor control and normal tissue complication. ${ }^{21}$

\section{Conflict of interest}

The authors declare that they have no conflicts of interest. The authors alone are responsible for the content and writing of the paper.

\section{References}

1. Verbakel WF, van Reij E, Ladenius-Lischer I, et al. Clinical application of a novel hybrid intensity-modulated radiotherapy technique for stage III lung cancer and dosimetric comparison with four other techniques. Int $J$ Radiat Oncol Biol Phys 2012; 83:e297-303.
2. Ong CL, Verbakel WF, Cuijpers JP, et al. Stereotactic radiotherapy for peripheral lung tumors: A comparison of volumetric modulated arc therapy with 3 other delivery techniques. Radiother Oncol 2010; 97:437-42.

3. Jiang X, Li T, Liu Y, Zhou L, et al. Planning analysis for locally advanced lung cancer: Dosimetric and efficiency comparisons between intensity-modulated radiotherapy (IMRT), single-arc/partial-arc volumetric modulated arc therapy (SA/PA-VMAT). Radiat Oncol 2011; 6:140.

4. Rao M, Yang W, Chen F, et al. Comparison of Elekta VMAT with helical tomotherapy and fixed field IMRT: Plan quality, delivery efficiency and accuracy. Med Phys 2010; 37:1350-9.

5. Holt A, van Vliet-Vroegindeweij C, Mans A, et al. Volumetric-modulated arc therapy for stereotactic body radiotherapy of lung tumors: A comparison with intensity-modulated radiotherapy techniques. Int J Radiat Oncol Biol Phys 2011; 81:1560-7.

6. Zhang GG, $\mathrm{Ku} \mathrm{L}$, Dilling TJ, et al. Volumetric modulated arc planning for lung stereotactic body radiotherapy using conventional and unflattened photon beams: A dosimetric comparison with 3D technique. Radiat Oncol 2011; 6:152.

7. Merrow CE, Wang IZ, Podgorsak MB. A dosimetric evaluation of VMAT for the treatment of non-small cell lung cancer. J Appl Clin Med Phys 2012; $14: 4110$.

8. Lu L. Dose calculation algorithms in external beam photon radiation therapy. Int J Cancer Ther Oncol 2013; 1:01025.

9. Rana S. Clinical dosimetric impact of Acuros XB and analytical anisotropic algorithm (AAA) on real lung cancer treatment plans: review. Int J Cancer Ther Oncol 2014; 2:02019.

10. Knöös T, Wieslander E, Cozzi L, et al. Comparison of dose calculation algorithms for treatment planning in external photon beam therapy for clinical situations. Phys Med Biol 2006; 51:5785-807.

11. Rana S, Rogers K, Pokharel S, Cheng C. Evaluation of Acuros XB algorithm based on RTOG 0813 dosimetric criteria for SBRT lung treatment with RapidArc. J Appl Clin Med Phys 2014; 15:4474.

12. Kathirvel M, Subramanian S, Clivio A, et al. Critical appraisal of the accuracy of Acuros-XB and Anisotropic Analytical Algorithm compared to measurement and calculations with the compass system in the delivery of RapidArc clinical plans. Radiat Oncol 2013; 8:140.

13. Ojala JJ, Kapanen MK, Hyödynmaa SJ, et al. Performance of dose calculation algorithms from three generations in lung SBRT: comparison with full Monte Carlo-based dose distributions. J Appl Clin Med Phys 2014; 15:4662. 
14. Fogliata A, Nicolini G, Clivio A, et al. Critical appraisal of Acuros XB and Anisotropic Analytic Algorithm dose calculation in advanced non-small-cell lung cancer treatments. Int J Radiat Oncol Biol Phys 2012; 83:1587-95.

15. Oyewale S. Dose prediction accuracy of collapsed cone convolution superposition algorithm in a multi-layer inhomogenous phantom. Int J Cancer Ther Oncol 2013; 1:01016.

16. Liu HW, Nugent Z, Clayton R, Dunscombe P, Lau $\mathrm{H}$, Khan R. Clinical impact of using the deterministic patient dose calculation algorithm Acuros XB for lung stereotactic body radiation therapy. Acta Oncol 2013. [Epub ahead of print]

17. Bush K, Gagne IM, Zavgorodni S, et al. Dosimetric validation of Acuros XB with Monte Carlo methods for photon dose calculations. Med Phys 2011; 38:2208-21.
18. Vassiliev ON, Wareing TA, et al. Validation of a new grid-based Boltzmann equation solver for dose calculation in radiotherapy with photon beams. Phys Med Biol 2010; 55:581-98.

19. Hawke S, Torrance A, Tremethick L. Evaluation of planned dosimetry when beam energies are substituted for a fraction of the treatment course. Int $J$ Cancer Ther Oncol 2013; 1:01014.

20. Pokharel S. Dosimetric impact of mixed-energy volumetric modulated arc therapy plans for high-risk prostate cancer. Int J Cancer Ther Oncol 2013; 1:01011.

21. Mavroidis P. Clinical implementation of radiobiological measures in treatment planning. Why has it taken so long? Int J Cancer Ther Oncol 2013; 1:01019. 